\title{
Visualising Museum Stories
}

\author{
Eoin Kilfeather \\ Dublin Institute of Technology \\ Dublin, Ireland \\ eoin.kilfeather@dmc.dit.ie
}

\begin{abstract}
Storytelling is fundamental to our ability to understand, and find meaning in, the world around us. Stories allow us to organise and share knowledge, and to identify the relationships between objects, events, and experiences. Within museums stories allow visitors to engage with collections and to gain meaningful understanding of museum objects. Stories transform a collection of objects into a meaningful knowledge structure that connects the objects and give them meaning.
\end{abstract}

This paper describes the Open Source Storyscope system that has been developed by the EU FP7 DECIPHER project. Storyscope's workspace and tools allow museum professionals and visitors to research, develop, and present stories that connect objects across museum collections. Storyscope has been built using advanced machine reasoning technologies that help the user to search for, organise and present resources from Collection Management Systems and from Linked Data sources.

A distinguishing feature of the system is the way in which semantically encoded "events", based on the CIDOC-CRM standard, are used to help visualise patterns of interconnectedness between, people, places and objects. The system uses an ontology to model the narrative while a semantic enrichment component helps users to identify story events "latent" in text resources and annotate those resources.

We describe Storyscope's rich HTML5 based User Interface which supports visualisation of event graphs. These event graphs can be used to traverse across museum objects in ways which might not be apparent through facet browsing, for example by connecting objects through shared events. The system also supports emplotment, by allowing users to make explicit authorial decisions about how events and objects connect together.

The project is supported by the EU FP7 Framework under grant FP7-270001-DECIPHER.

Narrative. HTML5. Collection management. Linked data.

\section{INTRODUCTION}

Although there is a vast and growing store of digital objects, most of them do not provide the information for explicit interpretation. On the other hand many cultural websites are extensive and elegant, but their narratives are 'burnt-in' and fixed by their authors: they can never be complete, and there is a good chance that the searcher's interests will not be fully met. Also, current systems are tied to the technology platforms for which they are authored.

The DECIPHER project has developed a new approach to the challenge of narrative construction in the museum, which uses knowledge visualization and an approach which seamlessly integrates research and narrative output. It combines much richer, event-based metadata with a narrative reasoning model. The reasoning engine, authoring environment and interfaces allow the user to present digital heritage objects as part of a coherent narrative, more directly related to individual searches and user contexts.

The system allows the user to interactively assemble, visualise and explore, not just collections of objects, but the knowledge structures that connect and give them meaning.

\section{MUSEUM PRACTICE}

The DECIPHER project was fortunate to have from the outset the involvement of museum professionals from two partner museums, the National Gallery of Ireland (NGI) and the Irish Museum of Modern Art (IMMA). These two museums have a combined annual visitor total of over a million and complemented each other in 
having collections which span Irish and European fine art on the one hand and Modern and contemporary art on the other. As the report on the research carried out by these two museums points out, the focus on museum practice has often been on the ideal (how museums should work) rather than on reality of how museums actually work. (Maguire et al. 2011). A key hypothesis of this research was that an important part of the work of these institutions is in fact the communication of knowledge through story (Bedford 2001).

In response the two museums carried out a programme of research into how their own, and other cultural institutions, actually generate narrative outputs. Rather than looking only at the work of the curator, the research looked at the role of other museum professionals such as educators, museum guides, and archivists.

In two workshops in 2011 the museums took two cases and examined the development of major exhibitions of work, one a review of modernism in Irish art titled The Moderns and the other a monographic exhibition titled Gabriel Metsu: Rediscovered Master of the Dutch Golden Age. These two exhibitions had recently closed and the museum staff involved addressed the processes involved in their genesis and implementation.

Presentations covered the research and curatorial design of the exhibition space; the design of education programmes and resources to complement the exhibition, such as teaching templates, student resources, audio guides and visitor booklets; touring exhibitions; and how the museum provides support for museum professionals and others to conduct research related to the exhibition.

Using these exhibitions as case studies the museums then looked critically at which workflows and processes, involving all the professional categories in the museum, contributed to the narrative outputs of those exhibitions. The result of that analysis was then used to define a broad ranging set of workflows and representative scenarios that were to be the subject of semantic formalisation. The principle output of this exercise was the development of a new model of museum workflows titled the Narrative Processes of Museum Professionals or NPMP.

\subsection{Formalising the NPMP}

Following from the work by the two museums in identifying the NPMP a semantic analysis of the two exhibition case studies was carried out by researchers in the Knowledge Media Institute of the Open University (OU). As an interim step to formalising these processes a report was produced which categorised the processes and mapped the NPMP processes into semantic concepts derived from structuralist approaches to narrative (Mulholland et al. 2011).

Four processes were identified, namely;

- Research: in which the person decides on a narrative concept and explores ideas around which to build a narrative.

- Dossier Building: comparable to the process of constructing a narrative via story and plot.

- Narrative Presentation: where the person prepares and publishes a narrative from the underlying story.

- Evaluation and Documentation: where additional material related to the narrative might become available through the public response to the narrative.

It is important to note that while the activities defined in the NPMP can be defined sequentially, in practice there is overlap in the individual activities across the top level processes. There is considerable level of task switching as the narrative construction switches between research and Dossier building phases. (Maguire et al. 2011). These processes and their association to the Storyscope software is show in Figure 1 below.

\subsection{The CURATE Ontology}

The semantic analysis carried out in conjunction with the museums produced a complex description of the narrative process within museums. The OU then proposed two working hypotheses to guide and test the interpretation of this data.

The first hypothesis was that museum narratives should have the sort of generic properties found in other types of narrative such as a novel or a film. In doing so Mullholland et al. (2012) drew on a number of structuralist theories of story (Hazel 2008). In these theories the story can be seen as the set of all events that can be told. A plot, by contrast, imposes a network of relationships on the events of the story and signals to the reader or viewer the events' roles, their importance in the overall story, and how they are related. The plot is an authorial and subjective interpretation of those events. A narrative is then a particular realisation of that story and plot, in some particular media and with some aesthetic consideration (Mulholland et al. 2012).

A second hypothesis underlying the ontology is that museum narratives are centred on artefacts (objects) that provide an important source of evidence. These objects become the subject of a narrative inquiry by the author in which a plot is essentially a hypothesis that is tested against the story. Story, plot and narrative therefore constitute 
a process rather than only associated types of description (Wolff et al. 2012).

The Curate ontology (http:/decipher.open.ac.uk/curate) is the formal representation of this approach to modelling heritage content by representing stories of both individual artefacts (objects) and exhibitions as a whole. The CIDOC CRM (http://www.cidoccrm.org/official release cidoc.html) and the DOLCE+DnS Ultralite (DUL, http://ontologydesignpatterns.org/ont/dul/DUL.owl) ontologies are used as the upper level ontologies for Curate.

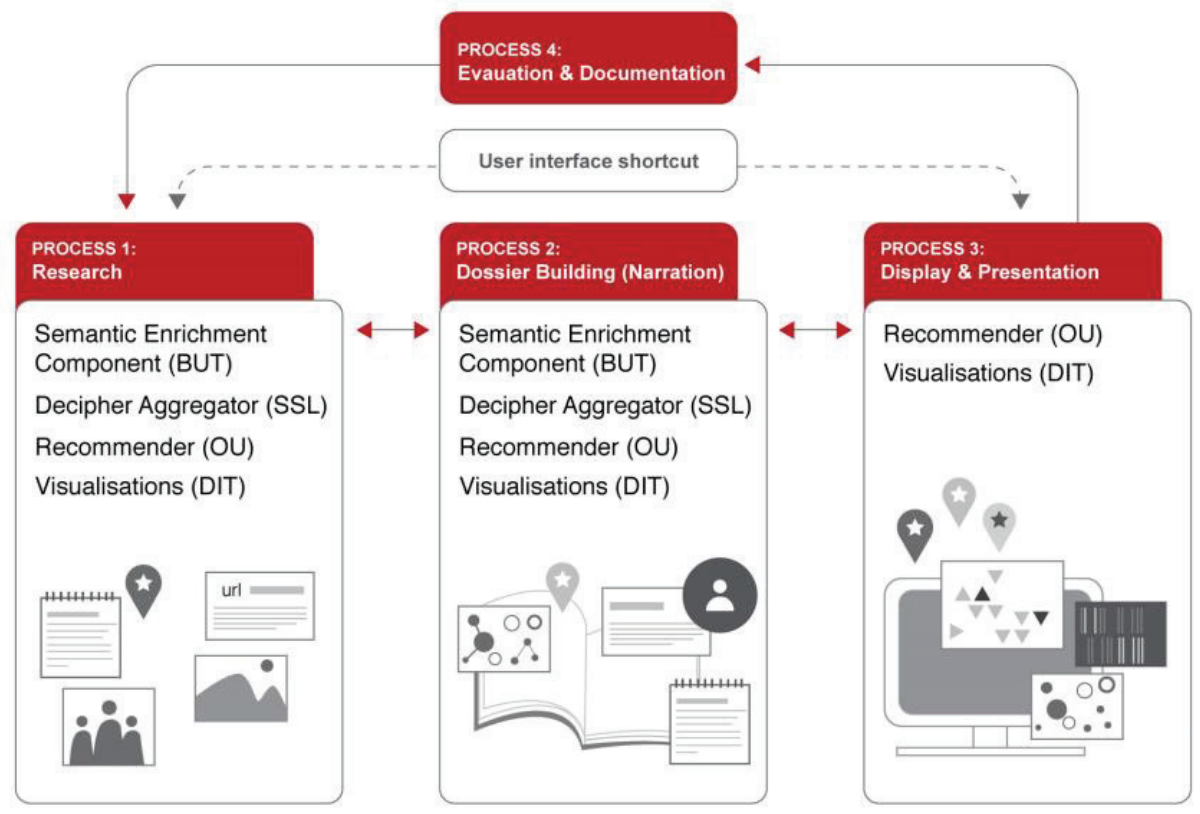

Figure 1: NPMP Processes and Storyscope Components

\subsection{Initial Prototype}

In the initial stages of the project the OU developed a prototype system titled StorySpace that allowed the Curate ontology to be tested and validated with museum professionals. Following initial trials this software was adopted by the consortium and used as the basis of the user facing components of the system. As the Storyspace name had previously been used by another software system the name was changed to Storyscope as part of a major refactoring of the software which took place in late 2012 (Kilfeather et al. 2012).

All the end-user interfaces are realised through the Storyscope system which is based on the Open Source Drupal architecture. The exception being a set of prototype Virtual Gallery narrative presentations that will be realised using narrative export templates and will utilise standards based web 3D techniques. The Storyscope system is described in greater detail in section 5 below.

\section{STORYSCOPE CONTENT}

The National Gallery of Ireland contributed content to a trial dataset of images (Objects) and Events. These objects were drawn from the Sir Denis Mahon Library and Archive, which was donated to the National Gallery of Ireland in 2010, and includes letters, photographs, catalogues, engravings, and books. This content added diversity within the dataset, and enabled the creation of more varied narratives. IMMA provided access to over 2000 images from its collection online. These images accounted for as much as $80 \%$ of the entire modern and contemporary collections.

The Alinari photographic archive in Florence (also a DECIPHER partner) provided a further small hand picked sample of 500 images for the dataset. Alinari worked with System Simulation (SSL), a partner, to programmatically provide an additional 20,000 images from its archives by selecting and harvesting content that include images of Art, Architecture, and Cityscapes from around the world. These datasets were used in formative trials of the prototype software that took place towards the end of 2012. 


\subsection{Content Aggregator}

The Content Aggregator (CA) is the ETL (Extract, Transform, Load) component of the DECIPHER system. It is responsible for ingesting structured metadata and digital assets from trusted sources, making them available to Storyscope components. It can ingest metadata supplied in a variety of formats and also ingest the associated digital assets. Where those assets have embedded metadata, the Aggregator can extract that metadata and pass it on for storage, indexing and the creation of Events (described in more detail in section 5 below).

The Aggregator stores the digital assets themselves in a reserved area of the host file system. As well as storing the submitted version of the asset, the Aggregator can create alternative versions, for instance with different resolutions or formats for use by the Storyscope system.

The CA can currently harvest the Europeana dataset and has an agreement with the VADS (http://www.vads.ac.uk/) resource for the visual arts to harvest its collection. These resources can be searched for within Storyscope and Objects of interest to the user imported for use.

\section{SEMANTIC ENRICHMENT}

The Semantic Enrichment Component (SEC) is a "backend" component used by Storyscope to process semi-structured or unstructured text. It has been developed by the Brno University of Technology (BUT), a DECIPHER partner. To do this it employs clustering, classification, and information extraction techniques to produce a variant of the text with semantically annotated entities and relations. Outputs can be transformed to an RDF description of events and other entities according to the CIDOC CRM. The SEC also supports an interactive service for annotating tracts of texts. Importantly it is also capable of making an estimates reliability of extracted semantic knowledge. (Smrz and Dytrych 2011)

The Content Aggregator can invoke the SEC. This would typically correspond to a situation in which the text harvested from web and Linked Data sources would be scheduled for processing by the SEC. The Similarity Recommender UI subcomponent of Storyscope also depends on the SEC functionality. It communicates with the Similarity Engine sub package and receives suggestions on potentially related entities to be presented to a user (Smrz et al. 2011).

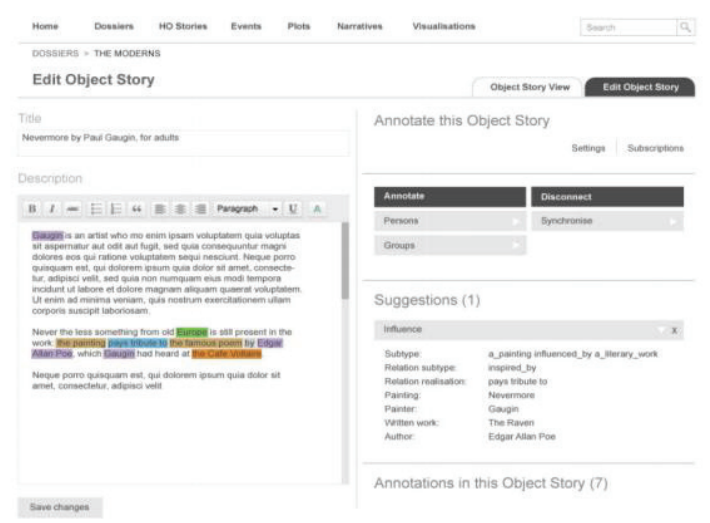

Figure 2: Semantic Enrichment UI prototype

\section{STORYSCOPE SYSTEM}

\subsection{Components}

The Storyscope system relies on a set of software components to function

- Storyscope Core: An authoring environment currently implemented with the Drupal 7 framework. This environment stores the content that the user needs to carry out research, compile resources, organise resources into compound objects and write narratives. It also holds (or links to) taxonomies and controlled vocabularies (currently drawn from Freebase entities) that help the user in selecting and entering data. This is the sole user-facing component; the other components provide services to this component.

- The Recommender Component: (RC) which stores a mapping of the content as RDF in a triple store and carries out machine reasoning. The purpose of the recommender is to suggest resources, such as Events or other Object Stories, to the user as she works.

- A Content Aggregator: (CA) which stores metadata and media related to museum collections which the user can search. Images and metadata of interest can be copied from this component into Drupal content by the user.

- A Semantic Enrichment Component: (SEC) which carries out Natural Language Processing of text sent to it by the user, harvested from open sources, or stored in the $\mathrm{CA}$. Its principle use is to analyse the text for entities and Events and to offer suggestions to automatically create or fill in the Properties of Events. 


\subsection{Storyscope concepts}

The system defines a number of concepts that determine the behavior of the User Interface.

Visibility: determines whether users other than the Owner can see the content. Visibility can only be un-set after which the content is visible to all other users in that Storyscope. This is to prevent the content being reused in another user's Dossier and then becoming invisible. Objects and References are always visible.

Context: The Dossier or Object Story that the user is currently working in. The context determines the actions available to the user, such as which Resources they can add to the context. The context can be determined by the URL (path) of any resource.

Resources: are all the Content Types that the user can use to carry out research and create narratives.

\subsection{Storyscope Actors}

User: a user of Storyscope is the owner of Resources and may edit and make visible Resources she owns. The user may make the resource visible to a Group or globally

Group: a group of Storyscope users who may collaborate in research and narrative authoring

\subsection{Storyscope content types}

Storyscope has the following content types.

- Source Events: Source events are an abstract representation of "something that happened" but with no explicit information about it. Source Events are used to link together Events which are different interpretations (with different properties and values) of the Source Event. Example: "World War II"

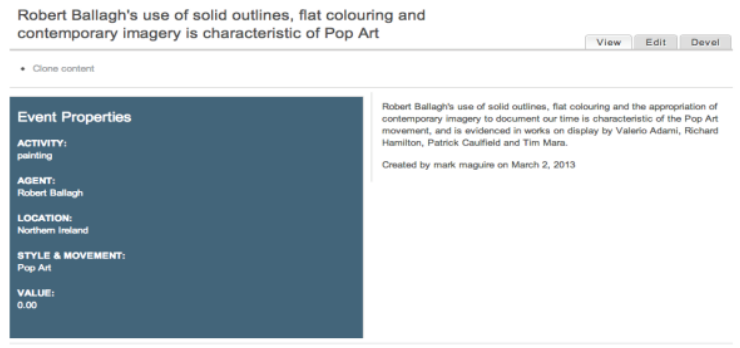

Figure 3: A Storyscope Event

- Events: Events are an interpretation of the parameters (properties and values) of an event, such as when it happened. Example: "World War II" - StartTime: 1939

- Objects: objects are media and metadata associated with an artefact such as a painting. Objects are always visible and can be used by any user but only edited by the owner.

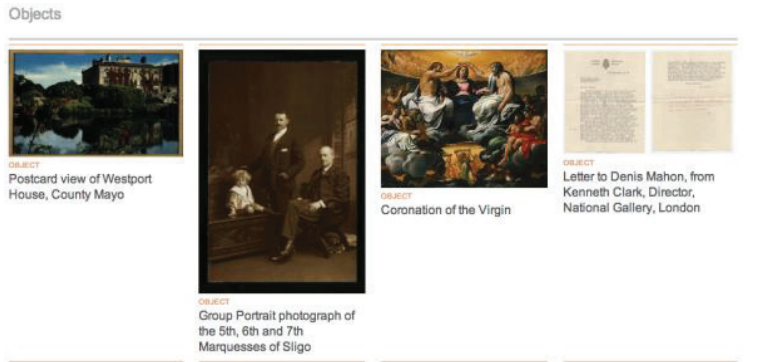

Figure 4: A set of Storyscope Objects

- References: references are media, descriptive text and resources locators relating to some external source of information such as a scholarly article. References are always visible and can be used by any user but only edited by the owner.

- Visualisations: a visualisation is a selection of Events within a Dossier or Object Story which is filtered and arranged based on the Events' properties These filter settings can be saved and given a Title. These saved settings may be visualised according to the Events' properties, for example: sorted on a grid according to property or property value; on a map or timeline by using the Location, Start Time and End Time properties.

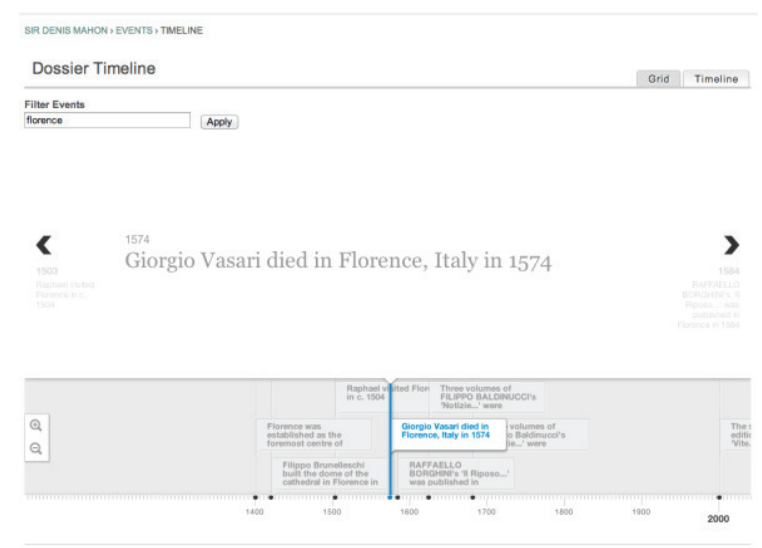

Figure 5: A Dossier timeline of Events

- Plots: a plot is a container for Plot Elements which the user groups together

- Plot Elements: a plot element is a container for a Plot Sets which a user groups together to explain some relationship between those Plot Sets

- Plot Set: holds a set of Events which are related. A plot set may be a source or a consequence of some other plot set. 
- Object Stories: object stories are a container for Objects, Events, Visualisations, and References usually relating to a particular artefact or related artefacts

- Dossiers: dossiers are a container for Object Stories, Events, Visualisations, and References usually related to a particular theme.

- Narratives: narratives are text or other media ordered and linked to some or all the resources from a Dossier or Object Story. It interprets the contents of a Dossier or Object story for a particular audience and / or delivery channel. It may be associated with a Plot.

- Narrative Element: a narrative element is a section or part of a narrative which may be linked to a corresponding Plot Set.

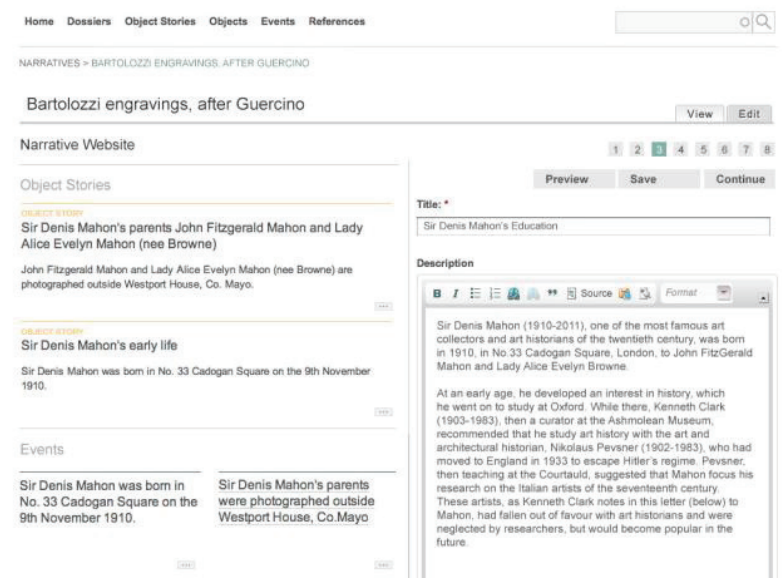

Figure 6: Prototype for editing a Narrative Element

\section{INTERFACES ARCHITECTURE}

Development of the User Interfaces in Storyscope is led by the Dublin Institute of Technology (DIT), the project coordinator. The interfaces (UI) rely on a complex framework of software modules, custom themes and CSS (SASS) mark-up. Within Storyscope the $\mathrm{UI}$ is realised through a custom theme called Storyscope Zen. The theme is a HTML5 based subtheme in Drupal 7, using a contributed theme called Zen as the basis. The CSS and mark-up are built using 'mobile first' principles, and a responsive layout that adapts to the width of the device that is viewing the site. (Kilfeather et al. 2012)

The CSS for the site is generated with the CSS framework Compass, which is built on the SASS3 language. SASS is a meta language for CSS that is used to describe the style of a document structurally and with more power than flat CSS allows. To ease the development of the intricate CSS required for responsive layouts, a Compass plugin called Zengrids is used. The Storyscope UI designs call for resources to be displayed in a number of different formats throughout. To achieve this, we have allowed nodes to be represented in a number of different build modes. These are principally "Full mode" and "Teaser" and these are built-in modes within Drupal. Other build modes such as 'thumbnail' and 'title only' are also supported.

Each Storyscope resource type is themed for each of these build modes. Using this framework allows for agile layout experiments in response to trial feedback and iterative UI refinements within the Storyscope. All layouts are developed, in so far as possible, in a generic manner to facilitate agile layout reconfiguration.

\section{SOFTWARE TRIALS}

The project is carrying out testing and evaluation of the Demonstrators, both in the laboratory and under field trial conditions, as the principal means of measuring the quality of the project outcomes. A Trial Scenarios and Evaluation Plan describe the procedures, criteria and metrics that are being applied. The Initial Trials and Formative Evaluation, was carried out in late 2012, and a full-blown Field Trials and User Evaluations has now begun and will continue until the winter of 2013.

The formative evaluation exercises were carried out under 'sandbox' conditions with a controlled dataset and small groups of users. This was done in two workshops using the formative software, which combined the initial prototypes of the reasoning engine and of the narrative interface. The resulting formative feedback was designed to provide qualitative information about interface, content, procedure and terminology issues. The initial trials were expected to involve professional users, although some input from visitor groups was anticipated. In the event a decision to port and refactor the software curtailed the formative trials before visitor feedback could be evaluated. The feedback gathered from the initial trials has been logged, analysed and fed back to the technical teams.

The result of the Initial trials has provided the starting point for the Field Trials. As noted above a significant outcome of the Formative Trials was a decision to port much of the Storyscope code from the Drupal 6 to the Drupal 7 architecture. This decision has allowed the software to utilise many more community-contributed modules and allowed for a much richer and expressive User Experience. 


\section{CURRENT AND FURTHER WORK}

The current focus of the project is on the development and testing of the Emplotment and Narrative publishing workflows.

The Storyscope system has recently been configured with an advanced SOLR indexing system to allow the user to easily navigate Dossier Events by "facet filtering" on Event properties such as "Agent" (the people or groups involved in an Event) or Activity (the "Verb") of the Event. Using these tools the user can manually identify the Events that might form the basis of a Plot Element a group of Events that are associated with each other.

On-going workshops are helping to refine the User Interfaces used in Plot construction. Work is also on-going to integrate the use of $\mathrm{Al}$ techniques for Event recommendation (from Linked Open Data sources), and also of entire Plot Elements based on the current contents of the user's Dossier. (Collins et al. 2012)

\section{REFERENCES}

Bedford, L. (2001) "Storytelling: The real work of museums." Curator: the museum journal 44(1): 27 34.

Collins, T. et al. (2012) Web supported emplotment: Using object and event descriptions to facilitate storytelling online and in galleries. Proceedings of the 3rd Annual ACM Web Science Conference, ACM.

Hazel, P. (2008) Narrative and New Media. Narrative in Interactive Learning Environments, Edinburgh.
Kilfeather, E. et al. (2012) DECIPHER: Interactive Interface Prototypes. http://decipherresearch.eu/deliverables-resources/D6.2.2.

Maguire, M. et al. (2011) DECIPHER: Museum Practices Report. http://decipherresearch.eu/deliverables-resources/D2.1.1.pdf.

Mulholland, P. et al. (2012) curate and storyspace: An ontology and web-based environment for describing curatorial narratives. The Semantic Web: Research and Applications, Springer: 748762.

Mulholland, P. et al. (2011) DECIPHER: Semantic processes workflow models and scenarios. http://decipher-research.eu/deliverablesresources/D2.1.2.

Smrz, P. and J. Dytrych (2011) Towards New Scholarly Communication: A Case Study of the 4A Framework. First Workshop on Semantic Publication (SePublica2011) 8 th Extended Semantic Web Conference Hersonissos, Crete, Greece, May 31, 2011.

Smrz, P. et al. (2011) DECIPHER: Relationship mining component. http://decipherresearch.eu/deliverables-resources/D4.2.1.

Wolff, A. et al. (2012) Storyspace: a story-driven approach for creating museum narratives. Proceedings of the 23rd ACM conference on Hypertext and social media, ACM. 